\title{
openheart Long-term follow-up of normal and structural heart ventricular tachycardia catheter ablation: real-world experience from a UK tertiary centre
}

\author{
Ahmed M Adlan, ${ }^{\oplus 1,2}$ Aruna Arujuna, ${ }^{2}$ Rory Dowd, ${ }^{2,3}$ Sajad Hayat, ${ }^{2}$ \\ Sandeep Panikker, ${ }^{2}$ Will Foster, ${ }^{2,4}$ Shamil Yusuf, ${ }^{2,3}$ Fraz Umar, ${ }^{5}$ Nicolas Lellouche, ${ }^{6}$ \\ Faizel Osman, ${ }^{\oplus, 8}$ Tarvinder Dhanjal $^{2}$
}

\begin{abstract}
- Additional material is published online only. To view please visit the journal online (http://dx.doi.org/10.1136/ openhrt-2018-000996).
\end{abstract}

To cite: Adlan AM, Arujuna A, Dowd $\mathrm{R}$, et al. Long-term followup of normal and structural heart ventricular tachycardia catheter ablation: real-world experience from a UK tertiary centre. Open Heart 2019;6:e000996. doi:10.1136/ openhrt-2018-000996

Received 20 December 2018 Revised 8 July 2019 Accepted 12 September 2019

Check for updates

(c) Author(s) (or their employer(s)) 2019. Re-use permitted under CC BY-NC. No commercial re-use. See rights and permissions. Published by BMJ.

For numbered affiliations see end of article.

Correspondence to Dr Tarvinder Dhanjal; tarv. dhanjal@uhcw.nhs.uk

\section{ABSTRACT}

Background Ventricular tachycardia (VT) is associated with increased morbidity and mortality. There is growing evidence for the effectiveness of catheter ablation in improving outcomes in patients with recurrent VT. Consequently the threshold for referral for VT ablation has fallen over recent years, resulting in increased number of procedures.

Objective To evaluate the effectiveness and safety of VT ablation in a real-world tertiary centre setting.

Methods This is a prospective analysis of all VT ablation cases performed at University Hospital Coventry. Followup data were obtained from review of electronic medical records and patient interview. The primary endpoint for normal heart VT was death, cardiovascular hospitalisation and VT recurrence, and for structural heart VT was arrhythmic death, VT storm ( $>3$ episodes within 24 hours) or appropriate shock.

Results Forty-seven patients underwent 53 procedures from January 2012 to January 2018. The mean age \pm SD was $57 \pm 15$ years, $68 \%$ were male, $81 \%$ were Caucasian and $66 \%$ were elective cases. The aetiology of VT included normal heart (49\%), ischaemic cardiomyopathy (ICM, 36\%), dilated cardiomyopathy (9\%), hypertrophic cardiomyopathy (4\%) and valvular heart disease (2\%). Procedural success occurred in $83 \%$, with six major complications. After a median follow-up of 231 days (lower quartile 133, upper quartile 631), the primary outcome occurred in $28 \%$ of patients. There were two non-arrhythmic deaths (4\%). At a median follow-up of 193 days (129-468), the primary outcome occurred in 19\% of patients with ICM, while VT storm/appropriate shocks occurred in three patients (17\%).

Conclusions Our real-world registry confirms that VT ablation is safe, and is associated with high acute procedural success and long-term outcomes comparable with randomised controlled studies.

\section{INTRODUCTION}

Ventricular tachycardia (VT) results in significant morbidity, and in patients with structural heart disease (SHD) (including prior myocardial infarction) is associated

\section{Key questions}

What is already known about this subject?

- Large multicentre trials show that catheter ablation for ventricular tachycardia (VT) is an effective and relatively safe therapy.

- The demand for VT ablation is growing due to increased survival of patients with myocardial infarction and a lower threshold for patient selection.

What does this study add?

- This real-world study confirms VT ablation is safe and associated with high acute procedural success and long-term outcomes comparable with randomised controlled studies.

How might this impact on clinical practice?

- This study provides evidence that an established ablation centre can deliver similar clinical outcomes (efficacy and safety) to those reported in large multicentre studies.

with increased risk of sudden cardiac death (SCD). ${ }^{1}{ }^{2}$ In myocardial infarction survivors the risk of SCD can be reduced with implantable cardioverter defibrillator (ICD) implantation. ${ }^{3}$ While ICDs are effective in terminating VT and aborting SCD, they do not prevent ventricular arrhythmias. Given that ICD shocks are associated with reduced quality of life and increased mortality, ${ }^{4}$ strategies are sought to prevent VT and minimise shocks. VT does not appear to significantly increase mortality in patients with structurally normal hearts, but can result in significant symptoms and cardiac dysfunction in patients with a high burden. ${ }^{5}$ Antiarrhythmic drugs (AADs) and catheter ablation have not been shown to improve mortality but do prevent recurrent VT in patients with or without ICDs. ${ }^{167}$ Despite their efficacy, AADs have an unfavourable safety profile, including proarrhythmia and extracardiac toxicity, 
particularly associated with long-term amiodarone use. ${ }^{6}$ Catheter ablation for VT directly targets and alters the arrhythmia substrate, ${ }^{8-12}$ reducing the need for AADs.

In patients with $\mathrm{SHD}$, catheter ablation has traditionally been reserved for patients with advanced cardiac disease and drug refractory VT. ${ }^{13}{ }^{14}$ However, in patients with ischaemic cardiomyopathy (ICM), accumulating evidence supports earlier intervention with catheter ablation. ${ }^{71516}$ Techniques for VT ablation have developed to allow for successful mapping and ablation of unstable VTs. ${ }^{10121617}$ As a result, the threshold to refer patients for VT ablation has fallen over recent years. Randomised control trials (RCTs) have recruited patients into large centres with extensive experience demonstrating the superiority of VT ablation over medical therapy; however, there are limited real-world data on the efficacy and safety of catheter ablation for VT in developing centres. We report our contemporary single-centre experience for VT ablation since the emergence of distinct patient selection criteria and advances in mapping and ablation technologies. In addition, we performed a substudy analysis on the efficacy and safety of patients with ICM compared with normal heart VT (nhVT). We hypothesised that our efficacy and safety would be comparable with that seen in contemporary RCTs.

\section{METHODS}

\section{Study design}

Detailed methodology can be found in the online supplementary material. Briefly, patients who underwent catheter ablation for VT performed at the University Hospital Coventry from January 2012 to January 2018 were prospectively entered into a registry. For nhVT (structurally normal heart on transthoracic echocardiogram or cardiac MRI, or cardiac dysfunction attributed to high premature ventricular contraction (PVC) burden), referral criteria included symptomatic VT despite medical therapy; intolerance to medication or patient preference against medication; PVC burden $>10 \%$; and cardiac dysfunction. For structural heart VT (shVT, ICM or non-ICM), indications included symptomatic VT despite medical therapy; three or more episodes of VT within 24 hours; at least three episodes of VT requiring antitachycardia pacing (ATP); or at least one appropriate defibrillator shock.

\section{Procedures}

All nhVT ablation procedures were performed under conscious sedation and selective shVT ablation procedures under general anaesthesia, where procedural risk was deemed high. Oral anticoagulation with warfarin was uninterrupted (target international normalised ratio 2.0-3.0). Direct oral anticoagulants were omitted on the day of the procedure. Anticoagulation was resumed the same day provided there were no significant bleeding events. When available a 12-lead ECG during VT was used to guide chamber mapping. Clinical VT induction was attempted with programmed electrical stimulation

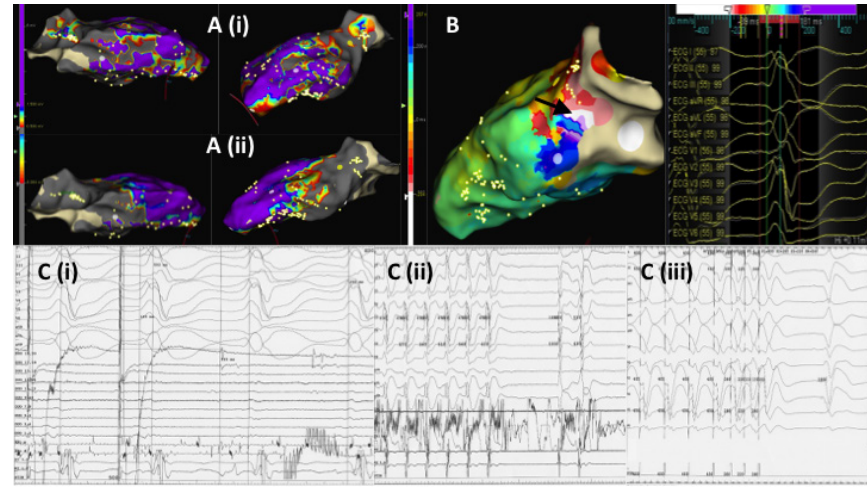

Figure 1 ICM ablation workflow demonstrating mapping strategies employed. (A) Endocardial substrate map with (i) bipolar scar definition $<1.5 \mathrm{mV}$ and dense scar $<0.5 \mathrm{mV}$ and (ii) unipolar scar definition $<8.0 \mathrm{mV}$. Septal and lateral views of the LV are shown, with grey scar extending from the lateral LV wall apical and inferior with the extensive basal septal scar. (B) Activation map of the right bundle branch block morphology VT TCL 544 ms. Mid-diastolic potentials were seen at the basal anterolateral LV (black arrow) shown as early meets late. (C) Entrainment mapping (i) from the basal anterolateral LV showing entrainment with concealed fusion (pace map 12/12), a postpacing interval (555 ms) minus TCL (544 ms) of $11 \mathrm{~ms}$ and stimulus to QRS of 103 ms. (C) Restoration of sinus rhythm during ablation (ii) with acute procedural success confirmed with non-inducibility (iii). ICM, ischaemic cardiomyopathy; LV, left ventricle; TCL, tachycardia cycle length; VT, ventricular tachycardia.

(PES). Patients were allowed to remain in VT during mapping and ablation if haemodynamically stable; otherwise VT was terminated with ATP or electrical direct current cardioversion. Electroanatomical substrate and activation mapping was performed using the EnSite NAVX/Velocity/Precision (Abbott Medical, Minneapolis, Minnesota) systems or CARTO 3 (Biosense Webster, Diamond Bar, California). All nhVT cases underwent pace mapping, ${ }^{11}$ and if VT was haemodynamically stable activation mapping was performed. For shVT a combination of substrate mapping, pace mapping and activation/entrainment mapping was performed, as shown in figure 1. The critical isthmus, late potentials, local abnormal ventricular activities (LAVA) potentials and decrementing early/late potentials were identified. ${ }^{8}$ Unstable VT mapping and ablation were performed using a substrate modification approach, ${ }^{12}{ }^{16}$ using a range of catheters including conventional and saline-irrigated ablation catheters ( $\leq 8 \mathrm{~mm}$ tips) (online supplementary table 1). Power, force-time integral, lesion size index and ablation index settings were at the operator's discretion. At the end of the procedure, standardised PES was performed. Complete acute procedural success was defined as termination of clinical VT with failure to induce clinical VT or VT with a longer tachycardia cycle length (TCL). To minimise follow-up bias, postprocedure ICD therapies (ATP/shock) were programmed according to a standardised protocol on the basis of the best evidence available. ${ }^{18} 19$ 


\section{Outcomes}

Follow-up was calculated from the first procedure until the last documented clinical contact after the VT ablation. The primary outcome for nhVT was a composite endpoint including all-cause death, cardiovascular hospitalisation and recurrent VT (defined as spontaneous sustained VT, or Holter evidence of PVC burden $>10 \%$ ). For shVT the primary outcome was a composite endpoint including arrhythmic death, appropriate shock or VT storm (defined as three or more documented VT episodes occurring within 24hours). Secondary outcomes included all-cause death, appropriate shock, recurrent VT, VT storm and cardiovascular hospitalisation. A substudy analysis was performed to compare VT ablation outcomes between patients with nhVT and ICM.

\section{Statistical analyses}

Statistical analyses were performed using SPSS V.22. Group differences were tested using Pearson's $\chi^{2}$ test for categorical variables, and independent t-tests or Wilcoxon test for continuous variables. $\mathrm{P}<0.05$ was statistically significant.

\section{RESULTS}

\section{Baseline demographics}

A total of 47 patients underwent 53 procedures between January 2012 and January 2018, with 43 cases (81\%) performed between March 2015 and January 2018 (figure 2). The mean age $\pm \mathrm{SD}$ was $57 \pm 15$ years. Patients were predominantly male $(68 \%)$ and Caucasian $(81 \%)$. The aetiology of VT included nhVT ( $\mathrm{n}=26,49 \%)$, including right ventricular outflow (RVOT) $(n=22)$, left ventricular outflow tract $(n=2)$ and fascicular VT $(n=2)$. shVT was common $(n=27,51 \%)$ and included ICM $(n=19)$, dilated cardiomyopathy $(n=5)$, hypertrophic cardiomyopathy $(n=2)$ and valvular heart disease $(n=1)$. Almost half of patients were referred locally $(n=25,47 \%)$, while the remainder were from our surrounding referral hospitals. Procedures were predominantly elective $(66 \%)$. The median time from index VT presentation to VT ablation was 318 (122-654) days. Baseline patient characteristics are shown in table 1 .

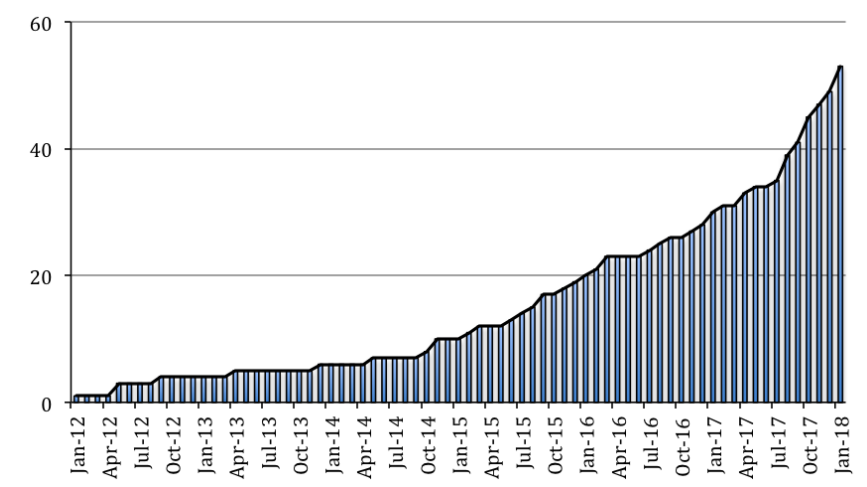

Figure 2 Cumulative frequency of ventricular tachycardia ablation procedures performed.
Hypertension, atrial fibrillation and heart failure were all more prevalent in the ICM group compared with nhVT. As expected, the left ventricular ejection fraction was significantly lower in the ICM group compared with the nhVT group $(29 \% \pm 1 \%$ vs $56 \% \pm 6 \% ; \mathrm{p}=0.011)$. None of the patients with nhVT had an ICD, whereas the majority of patients with ICM had defibrillators $(89 \%)$ and all had received therapies (94\% ATP, 76\% shocks). The most common antiarrhythmic agents included betablockers $(91 \%)$, amiodarone $(43 \%)$, mexiletine $(11 \%)$, verapamil $(8 \%)$ and flecainide $(2 \%)$. Only one patient with nhVT was treated with amiodarone compared with 17 of 19 patients with ICM $(89 \%)$. Mexiletine had been used in five patients with ICM and one patient with dilated cardiomyopathy. A significant proportion of patients were taking anticoagulation (39\%): warfarin (25\%) or apixaban $(11 \%)$. Significantly more patients were anticoagulated with ICM compared with nhVT $(\mathrm{p}=0.041)$.

\section{Procedural data}

A summary of the procedural data is shown in online supplementary table 1 . All nhVT cases were performed under local anaesthesia and conscious sedation, with only 4 of 19 ICM cases requiring general anaesthesia (21\%). The majority of patients had two or more clinical VTs $(87 \%)$, and most patients were in sinus rhythm at the start of the procedure $(93 \%)$. Clinical VT was inducible or spontaneous in all patients with nhVT and $58 \%$ of patients with ICM. VT was haemodynamically stable in all patients with nhVT compared with less than two-fifths of patients with ICM $(37 \%, \mathrm{p}<0.001)$. The Abbott Velocity/ Precision mapping system was used in nearly two-thirds of cases, with CARTO 3 in the remainder. The most common multipolar mapping catheters included Pentaray (32\%), HD linear Duodeca (21\%) and the Array (19\%).

Mapping was almost exclusively performed via the endocardial route $(98 \%)$. The most common mapping strategies included pace mapping (68\%), activation mapping (68\%) and substrate mapping (57\%); entrainment mapping was performed less commonly (11\%). In the ICM cohort all cases underwent substrate mapping, with additional $68 \%$ pace mapping, 22\% entrainment mapping and $47 \%$ activation mapping. Pace mapping (when used) was successful in achieving an 11/12 ECG match in $94 \%$ of cases. Ablation catheters used included SmartTouch (30\%), TactiCath (28\%) and M Therapy $(25 \%)$. For all cases the median radio frequency (RF) ablation time was 22 (IQR 11-48) min, while the median total procedural time was 200 (IQR 141-241) min. The median fluoroscopy time was 27 (19-34) $\mathrm{min}$. The nhVT group had shorter RF ablation time (14 vs $56 \mathrm{~min}$, $\mathrm{p}<0.001)$, overall procedural time $(142$ vs $240 \mathrm{~min}$, $\mathrm{p}<0.001$ ) and fluoroscopy times (21 vs $28 \mathrm{~min}, \mathrm{p}=0.028$ ) compared with ICM VT.

\section{Outcomes}

Acute procedural success occurred in $83 \%$ of all patients, with no significant difference between ICM and nhVT 


\section{Table 1 Baseline patient characteristics}

\begin{tabular}{|c|c|c|c|c|}
\hline & All & nhVT & ICM & $P$ value \\
\hline Procedures & 53 & 26 & 19 & \\
\hline Patients & 47 & 23 & 16 & \\
\hline Age, years & $57 \pm 15$ & $48 \pm 13$ & $70 \pm 9$ & $<0.001$ \\
\hline Sex: male & $36(68)$ & $15(58)$ & $14(74)$ & 0.268 \\
\hline Caucasian & $43(81)$ & $18(69)$ & $18(95)$ & 0.035 \\
\hline Asian & $3(6)$ & $8(31)$ & $1(5)$ & \\
\hline Hypertension & $14(26)$ & $2(8)$ & $10(53)$ & 0.002 \\
\hline Atrial fibrillation & $12(23)$ & $1(4)$ & $8(42)$ & 0.002 \\
\hline Diabetes & $4(8)$ & $1(4)$ & $2(11)$ & 0.375 \\
\hline Stroke & 0 & & & \\
\hline Left ventricular systolic dysfunction (LVEF $<40 \%$ ) & $21(40)$ & 0 & $15(79)$ & $<0.001$ \\
\hline LVEF, $\%$ & $43 \pm 16$ & $56 \pm 6$ & $29 \pm 11$ & 0.011 \\
\hline Wall motion abnormalities & $22(43)$ & $1(4)$ & $17(89)$ & $<0.001$ \\
\hline Previous VT ablation & $5(9)$ & $1(4)$ & $3(16)$ & 0.164 \\
\hline Implantable cardioverter defibrillator implanted & $24(45)$ & 0 & $17(89)$ & $<0.001$ \\
\hline \multicolumn{5}{|l|}{ Device therapies received } \\
\hline ATP & $22(92)$ & - & $16(94)$ & \\
\hline Shock & $16(67)$ & - & $13(76)$ & \\
\hline Beta-blocker & $48(91)$ & $23(88)$ & $19(100)$ & 0.125 \\
\hline Amiodarone & $23(43)$ & $1(4)$ & $17(89)$ & $<0.001$ \\
\hline Duration of amiodarone, median (IQR) months & $12(2-23)$ & 12 & $9(1-24)$ & \\
\hline Mexiletine & $6(11)$ & 0 & $5(26)$ & 0.006 \\
\hline Verapamil & $4(8)$ & $4(15)$ & 0 & 0.073 \\
\hline Flecainide & $2(4)$ & $2(8)$ & 0 & 0.216 \\
\hline Anticoagulation & $21(39)$ & $6(23)$ & $10(53)$ & 0.041 \\
\hline Warfarin & $13(25)$ & $5(19)$ & $7(37)$ & \\
\hline Apixaban & $6(11)$ & $1(4)$ & $3(16)$ & \\
\hline Dabigatran & $1(2)$ & 0 & 0 & \\
\hline Edoxaban & $1(2)$ & 0 & 0 & \\
\hline
\end{tabular}

Values expressed as mean \pm SD or frequency (\%) unless otherwise specified.

ATP, antitachycardia pacing; ICM, ischaemic cardiomyopathy; LVEF, left ventricular ejection fraction; nhVT, normal heart VT; VT, ventricular tachycardia.

( $84 \%$ vs $85 \%, p=0.970)$. In total, six patients underwent redo procedures. All patients $(n=47)$ were followed up for a median of 231 (133-631) days postprocedure. Sixteen patients with ICM were followed up for a median of 193 (129-468) days, and 24 patients with nhVT were followed up for 358 days ( $134-358$ days, $\mathrm{p}=0.205)$. The primary and secondary outcomes are shown in table 2 . The primary outcome occurred in 13 patients (28\%), with no significant difference between ICM and nhVT cases. Death occurred in two patients; however, these were non-arrhythmic. Three of 16 (19\%) patients suffered recurrent VT storm or appropriate shocks. Of the 13 patients with ICM who received shock therapy preprocedure, only 1 (8\%) patient received a recurrent shock. There were no significant differences in secondary outcomes between patients with ICM and nhVT. Procedural complication rates were low (11\%) and included three pericardial effusions not requiring intervention, one cardiac tamponade requiring pericardiocentesis, one cardiac perforation requiring emergency cardiac surgical repair with sternotomy and one groin haematoma managed conservatively.

\section{DISCUSSION}

The ventricular tachycardia ablation versus escalated antiarrhythmic drug therapy in ischemic heart disease (VANISH) trial demonstrated that catheter ablation in patients with drug refractory VT was effective in reducing the rate of VT storm and ICD shocks, with a reduction in the composite endpoint including death. ${ }^{7}$ Our real-world data show that catheter ablation for nhVT and shVT is 


\begin{tabular}{lllll}
\hline Table 2 & Outcomes & & & \\
\hline & All & nhVT & ICM & P value \\
\hline & 47 & 24 & 16 & \\
\hline $\begin{array}{l}\text { Primary outcome* } \\
\text { Secondary outcomes }\end{array}$ & $13(28)$ & $9(38)$ & $3(19)$ & 0.205 \\
\hline $\begin{array}{l}\text { Cardiovascular } \\
\text { hospitalisation }\end{array}$ & $10(21)$ & $5(21)$ & $5(31)$ & 0.456 \\
$\begin{array}{l}\text { Death } \\
\text { Recurrent VT/shock }\end{array}$ & $19(40)$ & $9(38)$ & $7(44)$ & 0.693 \\
\hline $\begin{array}{l}\text { VT storm } \dagger \\
\text { Appropriate shocksł }\end{array}$ & $4(17)$ & & $3(19)$ & \\
\hline
\end{tabular}

Values reported as frequency (\%).

${ }^{*}$ The primary outcome for nhVT was a composite of all-cause death, cardiovascular hospitalisation and recurrent VT, defined as sustained VT or PVC burden $>10 \%$. For shVT the primary outcome was a composite of arrhythmic death, VT storm ( $\geq 3 \mathrm{VT}$ episodes in 24 hours) and appropriate shocks.

†Reported as a percentage of patients with SHD. ‡Reported as a percentage of patients with ICDs.

ICDs, implantable cardioverter defibrillators; ICM, ischaemic cardiomyopathy; nhVT, normal heart VT; PVC, premature ventricular contraction; SHD, structural heart disease; shVT, structural heart VT; VT, ventricular tachycardia.

relatively safe, and is associated with a high rate of acute procedural success and long-term success. The outcome of shVT ablation in our study was comparable with the VANISH trial's primary outcome. In this multicentre RCT of 259 patients with ICM and recurrent VT despite AAD therapy, VT ablation was superior to escalating AAD therapy. ${ }^{7}$ In a meta-analysis of three RCTs including 346 patients, VT ablation was found to reduce ICD therapies, including shocks and electrical storm, with no overall effect on mortality. Furthermore time to VT recurrence was significantly delayed in patients undergoing catheter ablation. ${ }^{67}$

The development and widespread availability of ICDs have resulted in a marked change in the survival of patients with VT. ${ }^{3}$ Patients who formerly would have succumbed to SCD now survive to experience recurrent VT episodes and ICD therapies. ICD therapy occurs in approximately $40 \%$ of patients within the first year after ICD implant for secondary prevention ${ }^{2021}$ and in 5\%-18\% of patients within 1 year after ICD implantation for primary prevention. ${ }^{322}$ This, coupled with an increasing frequency of ICD implantation, ${ }^{23} 24$ has resulted in an increasing frequency of patients presenting with recurrent ICD shocks and the likelihood of a growing problem. Camm and Nisam ${ }^{23}$ showed that implant rates for ICDs in the USA increased exponentially from 2003, and after a lag of 4 years VT ablation rates have risen with a similar trend. ${ }^{14}$ UK data have shown an exponential rise in high energy implant rates from 2013, with 238 implants/million of the population in $2015 / 2016 .{ }^{24}$ Our registry data have shown a similar exponential rise in VT ablation cases since January 2016.

RCTs have recruited patients into large centres with extensive experience in VT ablation over several years. The
VANISH trial was a multicentre RCT conducted at 22 tertiary centres where VT ablation was routinely performed, with centres chosen to reflect 'a high level of competence in VT ablation technique'. We have shown that using the same patient selection criteria, an established regional centre can deliver a comparable clinical outcome of a relatively higher risk procedure with a safety profile comparable with nhVT ablation. The VANISH trial recruited patients treated with amiodarone for at least 3 months. Our cohort of patients with ICM included 15 of 16 (89\%) patients treated with amiodarone for at least 9 months. Qualifying episodes of VT in VANISH were required to be monomorphic and to have a cycle length greater than $240 \mathrm{~ms}$ (250 beats per minute), which was consistent with our cohort of patients with ICM $(379 \pm 19 \mathrm{~ms})$. In VANISH the primary outcome was a composite endpoint including death, VT storm or appropriate ICD shocks, which occurred less frequently in patients undergoing ablation versus patients having escalated AAD therapy ( $59.1 \%$ vs $68.5 \%$, HR 0.72 , $95 \%$ CI 0.53 to $0.98, p=0.04$ ). In our registry we report a primary outcome (arrhythmic death, VT storm or appropriate shocks) of $19 \%$ at a median of 6-month follow-up. When using the same primary outcome as in VANISH (all-cause death, VT storm or appropriate shocks) at 6 months which was approximately $20 \%$, we showed an event rate of $31 \%$. Two large prospective multicentre trials reported acute success rates (defined as elimination of all inducible VTs) of $41 \%$ and $49 \%$ with long-term rates of freedom from VT of $44 \%$ and $53 \%$, respectively. ${ }^{25}$ Our acute procedural endpoint performed in all patients with ICM demonstrated a procedural success rate of $84 \%$. A strength of our study was the definition of acute procedural success in ICM, based on data from Carbucicchio et $a .^{13}{ }^{13}$ It was shown that although elimination of all VTs (class A endpoint) provided the best outcome in terms of cardiac death, VT storm and any VT recurrence, the endpoint of clinical VT non-inducibility alone (class B endpoint) yielded a comparable cardiac death and recurrent VT storm primary outcome. The class B definition of success included residual VT with both longer and shorter cycle lengths to the clinical VT. Given that following ablation recurrent VT is unlikely to present with a shorter TCL, ${ }^{26}$ we believe that our definition of success was more robust than the class B definition, as it included patients with successful ablation of clinical VT and all VTs with a longer TCL. This is further supported by the heart center of leipzig ventricular tacycardia (HELP-VT) registry data, where higher VT recurrence rates were observed in the class B defined cohort. ${ }^{27}$

Our outcomes for nhVT were comparable with a large multicentre retrospective study of 1185 patients undergoing catheter ablation of PVCs recruited over a 9-year period. ${ }^{28}$ With similar baseline characteristics to our cohort, Latchamsetty et $a l^{28}$ reported an acute procedural success rate of $84 \%$ and a long-term success rate of $85 \%$ after a mean of 1.9 years of follow-up.

Our study was not designed or powered to detect differences in mapping or ablation strategies. Various strategies 
in VT ablation include targeting scar border zone with circumferential lines \pm dense scar lines, scar homogenisation, LAVA/late potential sites, critical isthmus targeting and scar dechannelling. The VISTA (ventricular tachycardia versus addition of substrate ablation on the long term success rate of VT ablation) trial compared clinical VT ablation versus substrate modification and showed better outcome in substrate-guided ablation. ${ }^{29}$ Both LAVA targeting ${ }^{9}$ and dechannelling ${ }^{8}$ have been shown to reduce recurrent VT. In haemodynamically unstable patients with VT, we routinely perform substrate-based ablation targeting border zones and LAVA/late potentials, and in more recent cases we adopted the dechannelling protocol which has yielded excellent non-inducibility endpoints. Technological advances in ablation lesion assessment over the course of the study resulted in increased use of force-time integral, lesion size index and ablation index in more recent cases.

A further strength of our study is that we reported rates of procedures performed under conscious sedation or general anaesthesia. Given the limited catheter laboratory time and general anaesthetic cover which most UK tertiary centres are challenged with, a predominate substrate mapping strategy has enabled the majority of our procedures to be safely performed under conscious sedation with procedural times reduced to less than 4 hours, requiring on average of $28 \mathrm{~min}$ of fluoroscopy and $56 \mathrm{~min}$ of ablation, comparable with the HELP-VT registry data. ${ }^{27}$

The high efficacy and safety outcomes reported in our real-world registry data reflect a combination of local expertise and a range of facilities in our established electrophysiology (EP) centre. Catheter ablation for VT can be complex and associated with an increased mortality risk when performed in low-volume or medium-volume centres $(<25$ postinfarct VT ablation procedures per year). ${ }^{14}$ In a European survey of 88 centres in 12 countries, approximately $22 \%$ and $14 \%$ of centres perform more than $50 \mathrm{VT}$ ablations per year for patients with normal heart and SHD, respectively. ${ }^{30}$ Readers are directed to a recent international expert consensus statement on catheter ablation of ventricular arrhythmias, which sets out requirements for operators and institutions to ensure ablations can be performed competently and safely. ${ }^{31}$ Briefly electrophysiologists should be adequately trained in mapping and ablation of complex ventricular arrhythmias. Institutions should have adequate facilities and personnel, including onsite interventional cardiologists (for coronary imaging); onsite cardiothoracic surgical backup for procedures requiring pericardial access in cases of the need for emergency sternotomy and cardiopulmonary bypass; and anaesthetic support.

\section{Study limitations}

This study is a single-centre, non-randomised cohort and represents the skills of six experienced operators. The mapping and ablation strategies employed were not predefined but rather reflected contemporary trends in techniques and technology. It is possible that the combination of technological advances and a 'learning curve' may account for better outcomes in the ICM group compared with the nhVT, which were predominantly performed in the early stages of our VT ablation service. Finally, in our study we did not include outcomes following repeat ablations for recurrent VT. During the study period we performed two ablation procedures in six patients, which may have resulted in improved outcomes than those reported in this present study.

\section{CONCLUSION}

These real-world registry data have demonstrated that catheter ablation for VT is safe and effective, with acute procedural and long-term outcomes comparable with RCTs. Furthermore, in our cohort we found similar safety and efficacy for VT ablation in patients with ICM and nhVT. Our study shows that using the same patient selection criteria as the VANISH trial, an established ablation centre can deliver an equivalent clinical outcome of a relatively higher risk procedure (ie, ICM VT catheter ablation) with a good safety profile comparable with nhVT.

\section{Author affiliations}

${ }^{1}$ Liverpool Heart and Chest Hospital NHS Foundation Trust, Liverpool, UK 2Department of Cardiology, University Hospital Coventry, Coventry, UK

${ }^{3}$ Department of Cardiology, Good Hope Hospital, University Hospitals Birmingham NHS Foundation Trust, Birmingham, UK

${ }^{4}$ Department of Cardiology, Worcestershire Royal Hospital, Worcester, UK

${ }^{5}$ University Hospital Coventry, Coventry, UK

${ }^{6}$ Hopital Henri Mondor, Paris, , France

${ }^{7}$ Cardiology, University Hospital Coventry, Coventry, UK

${ }^{8}$ Warwick Medical School, University of Warwick, Coventry, UK

Twitter Ahmed M Adlan @ahmed_adlan

Contributors TD and FO designed the study. TD, AMA, AA and RD collected the data. TD, FO, SH, SP, WF and SY recruited the patients and performed the procedures. AMA performed the statistical analysis and drafted the manuscript. All authors performed the critical review of the manuscript, including multiple drafts, and approved the final version.

Funding The authors have not declared a specific grant for this research from any funding agency in the public, commercial or not-for-profit sectors.

Competing interests None declared.

Patient consent for publication Not required.

Ethics approval The study applied the principles of the Declaration of Helsinki, with ethical approval obtained from our local audit and research department.

Provenance and peer review Not commissioned; externally peer reviewed.

Data availability statement Data are available upon reasonable request.

Open access This is an open access article distributed in accordance with the Creative Commons Attribution Non Commercial (CC BY-NC 4.0) license, which permits others to distribute, remix, adapt, build upon this work non-commercially, and license their derivative works on different terms, provided the original work is properly cited, appropriate credit is given, any changes made indicated, and the use is non-commercial. See: http://creativecommons.org/licenses/by-nc/4.0/.

\section{REFERENCES}

1. Al-Khatib SM, Stevenson WG, Ackerman MJ, et al. $2017 \mathrm{AHA}$ / ACC/HRS guideline for management of patients with ventricular arrhythmias and the prevention of sudden cardiac death: Executive summary: a report of the American College of 
Cardiology/American heart association Task force on clinical practice guidelines and the heart rhythm Society. Heart Rhythm 2018;15:e190-252.

2. Priori SG, Blomström-Lundqvist C, Mazzanti A, et al. 2015 ESC guidelines for the management of patients with ventricular arrhythmias and the prevention of sudden cardiac death: the task force for the management of patients with ventricular arrhythmias and the prevention of sudden cardiac death of the European Society of cardiology (ESC). endorsed by: association for European paediatric and congenital cardiology (AEPC). Eur Heart $J$ 2015;36:2793-867.

3. Bardy GH, Lee KL, Mark DB, et al. Amiodarone or an implantable cardioverter-defibrillator for congestive heart failure. N Engl J Med 2005;352:225-37.

4. Poole JE, Johnson GW, Hellkamp AS, et al. Prognostic importance of defibrillator shocks in patients with heart failure. $N$ Engl J Med 2008;359:1009-17.

5. Yarlagadda RK, Iwai S, Stein KM, et al. Reversal of cardiomyopathy in patients with repetitive monomorphic ventricular ectopy originating from the right ventricular outflow tract. Circulation 2005;112:1092-7.

6. Santangeli P, Muser D, Maeda S, et al. Comparative effectiveness of antiarrhythmic drugs and catheter ablation for the prevention of recurrent ventricular tachycardia in patients with implantable cardioverter-defibrillators: a systematic review and meta-analysis of randomized controlled trials. Heart Rhythm 2016;13:1552-9.

7. Sapp JL, Wells GA, Parkash R, et al. Ventricular tachycardia ablation versus escalation of antiarrhythmic drugs. N Engl J Med 2016;375:111-21.

8. Berruezo A, Fernández-Armenta J, Andreu D, et al. Scar dechanneling: new method for scar-related left ventricular tachycardia substrate ablation. Circ Arrhythm Electrophysiol 2015;8:326-36.

9. Jaïs P, Maury P, Khairy P, et al. Elimination of local abnormal ventricular activities: a new end point for substrate modification in patients with scar-related ventricular tachycardia. Circulation 2012;125:2184-96.

10. Soejima K, Suzuki M, Maisel WH, et al. Catheter ablation in patients with multiple and unstable ventricular tachycardias after myocardial infarction: short ablation lines guided by reentry circuit isthmuses and sinus rhythm mapping. Circulation 2001;104:664-9.

11. Stevenson WG, Khan $\mathrm{H}$, Sager $\mathrm{P}$, et al. Identification of reentry circuit sites during catheter mapping and radiofrequency ablation of ventricular tachycardia late after myocardial infarction. Circulation 1993;88:1647-70.

12. Tanner $\mathrm{H}$, Hindricks $\mathrm{G}$, Volkmer $\mathrm{M}$, et al. Catheter ablation of recurrent scar-related ventricular tachycardia using electroanatomical mapping and irrigated ablation technology: results of the prospective multicenter Euro-VT-study. J Cardiovasc Electrophysiol 2010;21:47-53.

13. Carbucicchio C, Santamaria M, Trevisi N, et al. Catheter ablation for the treatment of electrical storm in patients with implantable cardioverter-defibrillators: short- and long-term outcomes in a prospective single-center study. Circulation 2008;117:462-9.

14. Palaniswamy $C$, Kolte D, Harikrishnan $P$, et al. Catheter ablation of postinfarction ventricular tachycardia: ten-year trends in utilization, in-hospital complications, and in-hospital mortality in the United States. Heart Rhythm 2014;11:2056-63.

15. Atti V, Vuddanda V, Turagam MK, et al. Prophylactic catheter ablation of ventricular tachycardia in ischemic cardiomyopathy: a systematic review and meta-analysis of randomized controlled trials. J Interv Card Electrophysiol 2018;53:207-15.

16. Reddy VY, Reynolds MR, Neuzil P, et al. Prophylactic catheter ablation for the prevention of defibrillator therapy. N Engl J Med 2007;357:2657-65

17. Marchlinski FE, Callans DJ, Gottlieb CD, et al. Linear ablation lesions for control of unmappable ventricular tachycardia in patients with ischemic and nonischemic cardiomyopathy. Circulation 2000;101:1288-96.

18. Moss AJ, Schuger C, Beck CA, et al. Reduction in inappropriate therapy and mortality through ICD programming. N Engl J Med 2012;367:2275-83.

19. Wilkoff BL, Williamson BD, Stern RS, et al. Strategic programming of detection and therapy parameters in implantable cardioverterdefibrillators reduces shocks in primary prevention patients: results from the prepare (primary prevention parameters evaluation) study. $J$ Am Coll Cardiol 2008;52:541-50.

20. Schron EB, Exner DV, Yao Q, et al. Quality of life in the antiarrhythmics versus implantable defibrillators trial: impact of therapy and influence of adverse symptoms and defibrillator shocks. Circulation 2002;105:589-94.

21. Connolly SJ, Dorian P, Roberts RS, et al. Comparison of betablockers, amiodarone plus beta-blockers, or sotalol for prevention of shocks from implantable cardioverter defibrillators: the optic study: a randomized trial. JAMA 2006;295:165-71.

22. Al-Khatib SM, Sanders GD, Mark DB, et al. Implantable cardioverter defibrillators and cardiac resynchronization therapy in patients with left ventricular dysfunction: randomized trial evidence through 2004. Am Heart J 2005;149:1020-34.

23. John Camm A, Nisam S. European utilization of the implantable defibrillator: has 10 years changed the 'enigma'? Europace 2010;12:1063-9.

24. Cunningham $D$, Cunningham $M$, Akosua $D$. National audit of cardiac rhythm management devices April 2015 National Institute of cardiovascular outcomes research (NICOR); 2017: 1-28.

25. Stevenson WG, Wilber DJ, Natale A, et al. Irrigated radiofrequency catheter ablation guided by electroanatomic mapping for recurrent ventricular tachycardia after myocardial infarction: the multicenter thermocool ventricular tachycardia ablation trial. Circulation 2008;118:2773-82.

26. Yokokawa M, Desjardins B, Crawford T, et al. Reasons for recurrent ventricular tachycardia after catheter ablation of post-infarction ventricular tachycardia. J Am Coll Cardiol 2013;61:66-73.

27. Dinov B, Fiedler L, Schönbauer R, et al. Outcomes in catheter ablation of ventricular tachycardia in dilated nonischemic cardiomyopathy compared with ischemic cardiomyopathy: results from the prospective heart centre of Leipzig VT (HELP-VT) study. Circulation 2014;129:728-36.

28. Latchamsetty R, Yokokawa M, Morady F, et al. Multicenter Outcomes for Catheter Ablation of Idiopathic Premature Ventricular Complexes. JACC Clin Electrophysiol 2015;1:116-23.

29. Di Biase L, Burkhardt JD, Lakkireddy D, et al. Ablation of stable VTS versus substrate ablation in ischemic cardiomyopathy: the VISTA randomized multicenter trial. J Am Coll Cardiol 2015;66:2872-82.

30. Tilz RR, Lenarczyk R, Scherr D, et al. Management of ventricular tachycardia in the ablation era: results of the European heart rhythm association survey. Europace 2018;20:209-13.

31. Cronin EM, Bogun FM, Maury P, et al. 2019 HRS/EHRA/APHRS/ LAHRS expert consensus statement on catheter ablation of ventricular arrhythmias: Executive summary. Heart Rhythm 2019. 